\section{(1) \\ CrossMark}

\title{
Learning from registries in pulmonary arterial hypertension: pitfalls and recommendations
}

\author{
Katleen Swinnen ${ }^{1}$, Rozenn Quarck ${ }^{1,2}$, Laurent Godinas ${ }^{2}$, Catharina Belge ${ }^{1,2}$ and \\ Marion Delcroix (10 ${ }^{1,2}$
}

Affiliations: ${ }^{1}$ Respiratory Division, Dept of Chronic Diseases, Metabolism \& Ageing, KU Leuven - University of Leuven, Leuven, Belgium. ${ }^{2}$ Dept of Respiratory Diseases, University Hospitals Leuven, Leuven, Belgium.

Correspondence: Marion Delcroix, Dept of Respiratory Diseases, University Hospitals of Leuven, Herestraat 49, B-3000 Leuven, Belgium. E-mail: marion.delcroix@uzleuven.be

@ERSpublications

Registries are essential for tracing clinical evolution in PAH and implementing recommendations for practice. Modern management of registries should be further developed and extended, since many questions remain unanswered. http://bit.ly/2Y1umZK

Cite this article as: Swinnen K, Quarck R, Godinas L, et al. Learning from registries in pulmonary arterial hypertension: pitfalls and recommendations. Eur Respir Rev 2019; 28: 190050 [https://doi.org/10.1183/ 16000617.0050-2019].

ABSTRACT Pulmonary arterial hypertension is a rare and incurable chronic disease characterised by a progressive increase in pulmonary vascular resistance and right heart failure. Patient registries collecting observational data can be of great value in the understanding of clinical problems. While clinical trials provide data in selected patient populations, registries better depict real-life practice. This review aims to reflect the input of patient registries in the general knowledge of the disease. Advances in epidemiology of the different subgroups, including data on incidence and/or prevalence, increasing age at presentation and stagnating diagnostic delay are reported. The importance of haemodynamic definition criteria and cardiac comorbidities are underscored. The review also shows the major transformation that pulmonary arterial hypertension therapeutic management has undergone, with still insufficient use of combination therapies; consecutive improvement in outcome; upcoming evidence in disfavour of anticoagulation; and validity of the available risk-stratification tools derived from large registries. Product registries are also briefly presented. Finally, the benefits of registries and methodological aspects are discussed, including immortal time bias, registry data quality and recommendations from EU organisations (EUCERD and PARENT).

\section{Introduction}

Pulmonary arterial hypertension $(\mathrm{PAH})$ is a rare and incurable chronic disease characterised by progressive increase in pulmonary vascular resistance and right heart failure. PAH has different causes, it can be idiopathic, familial or associated with a number of conditions such as connective tissue disease, congenital heart disease, portal hypertension, HIV infection and exposure to toxins and drugs [1]. Despite considerable advances in the understanding of its pathophysiology and the development of several $\mathrm{PAH}$-specific therapies, the outcome of $\mathrm{PAH}$ remains poor.

To obtain comprehensive epidemiological data, numerous PAH registries have been set up at local, regional, national and international levels. Disease registries, which include patients based on diagnosis,

\section{This article has supplementary material available from err.ersjournals.com}

Provenance: Submitted article, peer reviewed.

Received: 07 May 2019 | Accepted after revision: 20 July 2019

Copyright CERS 2019. This article is open access and distributed under the terms of the Creative Commons Attribution Non-Commercial Licence 4.0. 
have served as an important source of data for characterising the various subtypes of PAH, assessing the burden and outcome of the disease, identifying risk factors, generating and validating hypothesis and measuring quality assurance and compliance to guidelines [2]. These registries also provide documentation of real-life treatment and on the transferability of randomised controlled trial (RCT) findings into practice. A distinction can be made between population-based registries, which refer to a geographically defined population and aim to register all cases in that population versus registries based on clinical centres or other organisations, where the population coverage may not be comprehensive [3].

During the Fifth World Symposium on Pulmonary Hypertension (PH) in 2013, McGoon et al. [2] addressed fundamental observations and three important biases in PAH registries: 1) survival bias, which is a sort of selection bias with overestimation of survival derived from a prevalent patient cohort if applied to newly diagnosed patients; 2) immortal time bias, which refers to a span of time in the observation or follow-up period of a cohort during which the outcome under study could not have occurred; and 3) case-wise deletion of patients due to missing data. The present review aims to integrate more recent data and discuss new insights of the disease.

\section{Chronology and characteristics of $\mathrm{PAH}$ registries}

In the field of PAH care, several disease registries have been established to collect data on clinical and haemodynamic parameters, prevalence and outcome (figure 1 and table 1) [4-6]. In the early 1980s, the National Institutes of Health (NIH) initiated the first large national registry in the USA to obtain data on natural history, treatment and pathogenesis of PH [4]. The NIH registry prospectively included 187 patients with primary PH (now reclassified as idiopathic PAH (IPAH), probably also including familial $\mathrm{PAH}$ and $\mathrm{PAH}$ associated with anorexigens) with a follow-up period up to 5 years. The main findings were as follows: 1) primary $\mathrm{PH}$ had a poor prognosis, as no PAH drugs were available at that time; and 2) prognosis was related to haemodynamic severity [7]. Two decades later, the Pulmonary Hypertension Connection registry was initiated in three university medical centres in Chicago (IL, USA), collecting data of patients with PAH diagnosed between 1982 and 2004 (retrospective cohort) and between 2004 and 2007 (prospective cohort), with the aim of providing contemporary survival data in a large number of patients with PAH $(n=576)$ [6]. Meanwhile, the French Network on PAH was initiated in 2002, as a national prospective registry [8]. This network collected data from 674 patients in 1 year and described patient characteristics and 1- and 3-year survival. The French investigators were the first to introduce the distinction between "incident" patients, who had just received the diagnosis, and "prevalent" patients, who had previously received the diagnosis and who entered the registry when returning for follow-up visit. In

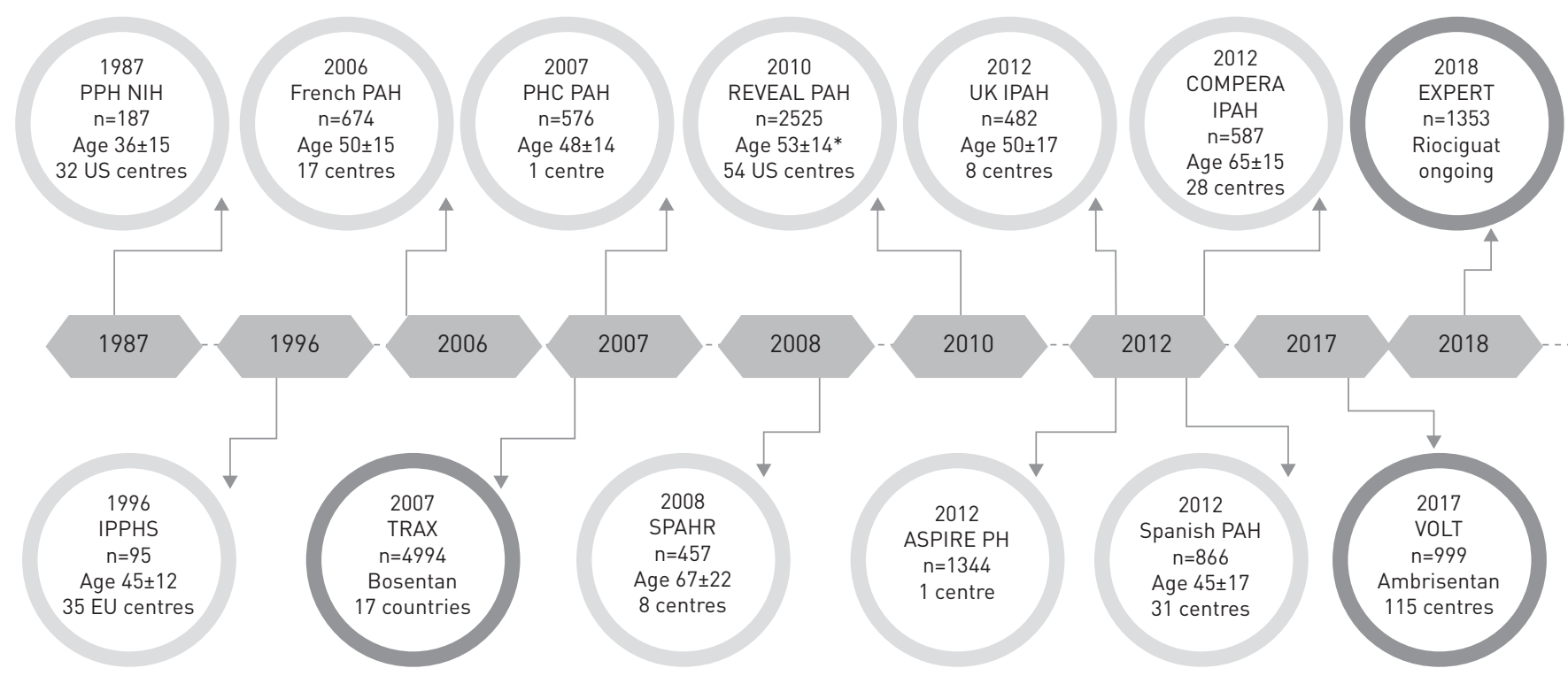

FIGURE 1 Timeline of pulmonary arterial hypertension (PAH) registries and their characteristics (list is not exhaustive). Darker circles indicate product registries as opposed to disease registries. PPH: primary pulmonary hypertension; NIH: National Institutes of Health; PHC: Pulmonary Hypertension Connection; IPAH: idiopathic PAH; IPPHS: International Primary Pulmonary Hypertension Study group; TRAX: Tracleer Excellence Post Marketing Surveillance Programme; SPAHR: Swedish Pulmonary Arterial Hypertension Registry; REVEAL: Registry to Evaluate Early and Long-Term Pulmonary Arterial Hypertension; ASPIRE: Assessing the Spectrum of Pulmonary Hypertension Identified at a Referral Centre; PH: pulmonary hypertension; COMPERA: Comparative, Prospective Registry of Newly Initiated Therapies for Pulmonary Hypertension; VOLT: the Volibris Tracking Study; EXPERT: Exposure Registry Riociguat in Patients with Pulmonary Hypertension. *: same mean age in IPAH patients. 
TABLE 1 Overview of data collected in pulmonary hypertension (PH) registries

\begin{tabular}{|c|c|c|c|c|c|c|c|c|c|c|}
\hline & $\begin{array}{c}\text { NIH } \\
1987\end{array}$ & $\begin{array}{c}\text { French } \\
2006\end{array}$ & $\begin{array}{c}\text { Swedish } \\
2007\end{array}$ & $\begin{array}{l}\text { PHC } \\
2007\end{array}$ & $\begin{array}{c}\text { Scottish } \\
2007\end{array}$ & $\begin{array}{c}\text { REVEAL } \\
2010\end{array}$ & $\begin{array}{l}\text { ASPIRE } \\
2012\end{array}$ & $\begin{array}{c}\text { UK } \\
2012\end{array}$ & $\begin{array}{c}\text { Spanish } \\
2012\end{array}$ & $\begin{array}{c}\text { COMPERA } \\
2012\end{array}$ \\
\hline $\begin{array}{l}\text { Incidence/ } \\
\text { prevalence }\end{array}$ & - & $x$ & $x$ & - & $x$ & $x$ & - & $x$ & $x$ & - \\
\hline PH (sub)groups & - & $x$ & - & - & $x$ & $\mathrm{x}$ & $x$ & - & $x$ & - \\
\hline Demographics & $x$ & $x$ & $x$ & $x$ & - & $x$ & $x$ & $x$ & $x$ & $x$ \\
\hline Symptoms & $x$ & $x$ & - & - & - & $x$ & - & $x$ & $x$ & - \\
\hline $\begin{array}{c}\text { Haemodynamic } \\
\text { parameters }\end{array}$ & $x$ & $x$ & $x$ & $x$ & - & $\mathrm{x}$ & $x$ & $x$ & $x$ & $x$ \\
\hline Treatment practices & - & $x$ & $x$ & $x$ & - & $x$ & $x$ & $x$ & - & $x$ \\
\hline $\begin{array}{l}\text { Outcome and } \\
\text { predictors }\end{array}$ & $x$ & $x$ & $x$ & $\mathrm{x}$ & - & $\mathrm{x}$ & $\mathrm{x}$ & $\mathrm{x}$ & $x$ & $x$ \\
\hline Oral anticoagulation & - & - & - & $x$ & - & $x$ & - & - & - & $x$ \\
\hline
\end{tabular}

NIH: National Institutes of Health; PHC: Pulmonary Hypertension Connection; REVEAL: Registry to Evaluate Early and Long-Term Pulmonary Arterial Hypertension; ASPIRE: Assessing the Spectrum of Pulmonary Hypertension Identified at a Referral Centre; COMPERA: Comparative, Prospective Registry of Newly Initiated Therapies for Pulmonary Hypertension; PFT: pulmonary function test.

2006, the Registry to Evaluate Early and Long-term PAH disease management (REVEAL) was started in the USA [9]. The REVEAL registry was established to provide updated characteristics of incident and prevalent patients with $\mathrm{PAH}$ and to improve diagnosis, treatment and $\mathrm{PH}$ management. The haemodynamic inclusion criteria were expanded as compared with the traditional PAH definition, with a pulmonary arterial wedge pressure (PAWP) up to $18 \mathrm{mmHg}$. Over an 18-month period, 54 US centres enrolled a total of 2967 patients. In addition, a registry was conducted in the UK, concentrating on a purely incident cohort of patients with PAH [10]. Between 2001 and 2009, 482 incident patients were included, which was a major contribution for the knowledge on incident and treatment-naive cases with $\mathrm{PAH}$ [10]. A Spanish registry of PAH was also launched and included 866 patients from referral centres and community hospitals in Spain over a 10-year period between 1998 and 2008 [11]. This registry provided specific data on the association of PAH with toxic oil syndrome. Finally, starting as a German registry, the Comparative Prospective Registry of Newly Initiated Therapies for Pulmonary Hypertension (COMPERA), initiated in 2007 and still ongoing, included patients from Austria, Belgium, Germany, Italy, the Netherlands, Switzerland and the UK, with any type of $\mathrm{PH}$ and receiving specific $\mathrm{PAH}$ medical therapies [12]. As of July 2018, over 8700 patients have been enrolled. Furthermore, the Swedish Pulmonary Arterial Hypertension Registry (SPAHR) was initiated in 2000 to collect ongoing data from patients [5]. The eight Swedish PAH centres participating in the registry have an estimated coverage of 90\% of all patients with PAH. Additionally, a single-centre registry Assessing the Spectrum of Pulmonary hypertension Identified at a Referral centre (ASPIRE) is the first and only registry presenting epidemiological data of patients from the five PH subgroups [13]. Until 2012, 1344 treatment-naïve patients have been enrolled over a 9-year period with a mean follow-up period of 2.9 years.

Besides disease registries, product registries gathered patient data in order to identify potential safety concerns associated with the acute and chronic use of drugs. Interestingly, these post-marketing pharmacovigilance studies also offered some epidemiological data [14-16]. Based on the suspicion of appetite suppressants displaying an increased risk of $\mathrm{PH}$, a landmark prospective case-control study from the International Primary Pulmonary Hypertension Study group (IPPHS) conducted in France, Belgium, the UK and the Netherlands was published in 1996. It demonstrated a strong causal relationship between anorectic drugs and $\mathrm{PH}$ [17]. Surprisingly, these results did not prevent the approval of dexfenfluramine for the treatment of obesity by the US Food and Drug Administration [18, 19]. In 2002, the dual endothelin receptor antagonist bosentan was approved as the first oral drug for PAH. Due to an increased rate of liver enzyme elevation in patients treated with bosentan, the Tracleer Excellence Post-Marketing Surveillance (TRAX) programme was started [14]. Between May 2002 and November 2004, almost 5000 patients were enrolled. The post-marketing surveillance confirmed that the incidence and severity of increased liver enzymes in clinical practice (annual rate 10\%) was similar to that reported in the registration trials $[20,21]$. The Volibris Tracking (VOLT) study was also designed to better characterise the long-term safety profile of ambrisentan, a type A endothelin receptor antagonist, and indicated no new ambrisentan-related risk of harm compared with those found in the clinical trials, including increased liver 
enzymes (annual rate 2\%), oedema (25\%) and anaemia (14\%) [15]. In 2014, riociguat, a soluble guanylate cyclase stimulator, was approved as a novel therapy for PAH and chronic thromboembolic $\mathrm{PH}$. In agreement with the regulatory guidance for safety, the EXPERT (Exposure Registry Riociguat in patients with PH) study was designed and recruited 1316 patients (ClinicalTrials.gov, identifier NCT02092818) [16]. Concurrently, the Opsumit Users Registry (OPUS; ClinicalTrials.gov, identifier NCT02126943), started in 2014 in the USA, aimed to evaluate the safety profile of the endothelin receptor antagonist, macitentan. Of note, this registry included a more heterogeneous and older population compared with RCTs [22]. Finally, SPHERE (Selexipag: the Users Drug Registry; ClinicalTrials.gov, identifier NCT03278002) is a US multicentre, prospective, real-world, observational drug registry enrolling patients treated with selexipag since November 2016 [23]. In addition, the EXPOSURE (European Observational Study of Uptravi in Real-Life) post-authorisation safety study started in 2017 and enrolled a European cohort of patients with $\mathrm{PAH}$ newly treated with either selexipag or any other PAH-specific therapy.

As illustrated above, registries may have multiple purposes and integrate data from various sources. Having a clear purpose enables the evaluation of whether the registry is capturing the information of interest. Attempts to be all inclusive may add cost but not value [3]. Most registries included data for analysis of the demographics, haemodynamics, outcomes and predictors for outcome. However, some also provide detailed data on incidence and/or prevalence, $\mathrm{PH}$ (sub)groups, symptoms and diagnostic delay, pulmonary function tests and treatment practices [7-13]. Only a few registries gathered data on the use of anticoagulants $[9,12,24]$. The REVEAL study is the only registry that also included patients with a PAWP between 16 and $18 \mathrm{mmHg}$, which is above currently accepted PAH diagnostic criteria [9]. Table 1 provides an overview of the data collected in each registry. These specific aspects are discussed in the following sections.

\section{Incidence and prevalence of PAH and IPAH}

The epidemiology of PAH displays some variation when comparing registries, probably related to selection biases. The incidence of PAH ranged from 2.0 cases per million population in the USA to 7.6 cases per million population in Scotland $[9,25]$. For IPAH, a sporadic disease with neither a family history nor an identified risk factor, a range of incidence is reported from 0.9 cases per million population in the USA to 2.6 cases per million population in Scotland (table 2) $[9,25]$.

The first insights in incidence and prevalence of PAH came from the US NIH registry published in 1987 [4]. Based on data of 187 enrolled patients with a mean age of 36 years, the annual incidence of IPAH was estimated at 1-2 cases per million population. This was confirmed in 1996, with an incidence of 1.7 cases per million in the Belgian population [17]. Similar figures were observed in the French and Spanish registries (table 2) $[8,11]$. Of note, much higher incidence and prevalence were observed in the Scottish hospitalisation registry, based on PAH hospitalisations in the period from 1986 to 2001 [13]. This unique population-based study suggested that previous reports issued from specialised centres could have underestimated the true incidence and prevalence of PAH. This hypothesis was recently supported by the Swedish registry data, showing an incidence of 5 patients and a prevalence of 25 patients with IPAH and heritable PAH (HPAH) per million inhabitants [5]. Overall, based on the published data of the past

\begin{tabular}{|c|c|c|c|c|}
\hline Registry & [Ref.] & & Incidence per $10^{6}$ population & Prevalence per $10^{6}$ population \\
\hline IPPHS & [17] & IPAH & 1.7 & \\
\hline French & [8] & IPAH & 1.0 & 5.9 \\
\hline French & {$[8]$} & IPAH & 2.4 & $15\left(4-29^{\#}\right)$ \\
\hline Scottish & [25] & $\mathrm{IPAH}$ & 2.6 & 9 \\
\hline Scottish & [25] & $\mathrm{IPAH}$ & 7.6 & 26 (52 $)$ \\
\hline REVEAL & [9] & IPAH & 0.9 & \\
\hline REVEAL & [9] & IPAH & 2.0 & 10.6 \\
\hline UK & [10] & $\mathrm{IPAH}$ & 1.1 & 6.6 \\
\hline Spanish & [11] & $\mathrm{IPAH}$ & 1.2 & 5.6 \\
\hline Spanish & [11] & $\mathrm{PAH}$ & 3.7 & 16 \\
\hline Swedish & [5] & IPAH & 5 & 25 \\
\hline
\end{tabular}

Data are presented as annual cases per million population for incidence and prevalence. IPAH: idiopathic pulmonary arterial hypertension; PAH: pulmonary arterial hypertension; REVEAL: Registry to Evaluate Early and Long-Term Pulmonary Arterial Hypertension. " : regional differences; ": Scottish hospitalisation registry. 
20 years, a trend of an increasing number of patients with PAH can be observed. Several potential factors could be responsible for the discrepancies in PAH epidemiology across registries. These include the quality of the data, the inclusion period and the geographic area. In addition, variations in ethnical background might be associated with genetic differences. The exposure to drugs may also contribute to changes in the occurrence of risk factors across registries. The availability of pharmacological treatment and more widespread availability and access to echocardiography may also account for variations in trends between studies. Differences in prevalence between the various parts of the world may also result from disparities in survival and recovery as well as in incidence. Last but not least, patients with PH due to heart failure with preserved ejection fraction might have been unintentionally included in many registries since they were not systematically excluded from enrolment other than with PAWP. In the future, one might advocate the collection of more systematic population-based data in order to improve our understanding of the disease epidemiology.

In particular, the current largest PH epidemiologic study included over 50000 individuals based on linked databases of universal coverage health service records comprising drug dispensing data, emergency, hospitalisation and ambulatory care data, and demographic information including mortality [26]. The use of health administrative "big data" has many potential upsides and limitations for rare diseases and there have been several recent studies that highlight methodologies [27, 28]. The major obstacle and source of bias is the lack of specificity of diagnosis codes commonly used in reimbursement system databases, such as the International Classification of Diseases, 9th Revision, which do not align with the updated $\mathrm{PH}$ classification [1]. Different methodological algorithms have been proposed combining $\mathrm{PH}$-related diagnosis codes together with PAH-specific medications and right heart catheterisation procedure codes, but few of them have been validated against electronic medical records [27, 28]. Linking existing PAH registries with health administrative data or using registries to validate health administrative data algorithms would be another way to move forward.

\section{Distribution in PAH subgroups}

Data regarding the epidemiology of $\mathrm{PAH}$ associated with other conditions are scarce and vary greatly across the different registries. Most patients are diagnosed with IPAH, HPAH or drug-induced PAH. The term HPAH includes IPAH with germline mutations and familial cases with or without identified mutations. They represent 3-4\% of the patients with PAH (French and US registries: $2.7 \%$ and: $3.9 \%$, respectively $[8,9])$. The proportion of drug- and toxin-induced $\mathrm{PAH}$ varies greatly from country to country depending on the exposure $[8,9,17]$.

PAH associated with congenital heart disease (CHD) is highly prevalent. It represents $11.3 \%$ of the French cohort, and $16 \%$ of the Spanish cohort, with a prevalence of 2.3 and 3 per million, respectively $[8,11]$. In the Scottish registry, the prevalence was much higher, reaching 7 cases per million [13]. According to the Dutch Congenital Corvitia (CONCOR) registry, lifetime risk of PAH after shunt closure may be much higher than previously thought [29]. While the risk of PH immediately after closure was only $2.1 \%$, the risk increased throughout follow-up, increasing $>15 \% 50$ years after closure. PAH also developed in $4.1 \%$ of patients who underwent closure of an atrium septum defect prior to the age of 25 years, a group previously thought to be at very low risk [29]. PAH-CHD may be underdiagnosed due to a low interaction between the $\mathrm{PH}$ centres and the physicians caring for patients with $\mathrm{CHD}$ [11]. PAH associated with connective tissue disease (CTD) represents another important subgroup. In France, a prevalence of 2.3 cases per million population was reported for PAH-CTD and the incidence was 0.4 per million. A similar prevalence of 2.5 per million was found in Spain $[8,11]$. In the Scottish registry, once again, the prevalence was much higher, reaching 10 cases per million with an incidence of 2.8 cases per million [13]. $\mathrm{PAH}$ due to portal hypertension is much less common, although it is still more frequent than PAH associated with HIV infection and PAH-schistosomiasis. PAH-HIV infection remains rare with an estimated prevalence of $0.5 \%$ of patients with HIV infection in developing countries [30,31]. In addition, the incidence of PAH-HIV is regularly decreasing, consequently to a better control of HIV infection [30]. Similarly, in the COMPERA registry, more patients had PAH-CTD and PAH-CHD, followed by a smaller percentage of the patients having PAH due to portal hypertension and PAH-HIV [12]. Interestingly, the distribution of $\mathrm{PH}$ associated with other conditions was not different in tertiary hospitals compared with a referral centre in the UK [13]. While schistosomiasis is one of the most common infections in the world, schistosomiasis-associated PAH is understudied and, disproportionally, little information exists on demographics and epidemiology of these patients. Up to $5 \%$ of the patients with the hepatosplenic form of the disease may develop PAH [32,33]. Additionally, pulmonary veno-occlusive disease (PVOD) is often misclassified as IPAH and, therefore, the true incidence and prevalence is still unknown and probably underestimated. It is currently accepted that PVOD represents $10 \%$ of the cases of PAH initially considered as idiopathic or heritable, thus suggesting an incidence rate of 0.5 cases per million per year in 
the French population [34, 35]. The prevalence of PVOD in the Spanish registry was 0.16 cases per million population [11].

Recently, a large multicentre study was initiated to explore the racial and ethnic differences in presentation, severity and treatment of patients with PAH [36]. An ongoing NIH-funded registry of patients with PAH enrolled from 34 US centres with clinical data, banked biological samples and genetic data from a PAH biobank, showed that African-American and Hispanic patients are more likely to present with $\mathrm{PAH}$ associated with $\mathrm{CTD}$ or $\mathrm{CHD}$, compared with non-Hispanic white patients [36]. Considering these issues, further studies are required to characterise the role of ethnicity in the prevalence, presentation and outcomes of $\mathrm{PH}$.

\section{Changing demographics of patients with PAH}

With the emergence of effective therapies for $\mathrm{PAH}$, the disease awareness has risen among both patients and physicians. Concomitantly, the population of most Western countries is ageing. Consequently, recent data from Europe and the USA suggest that the demographics of patients diagnosed with IPAH are changing. In the past, most patients were young with a mean age of 36 years at diagnosis, predominantly female and had IPAH [4]. In more recent registries, the median age is 67 years in the Swedish registry and 71 years in the COMPERA registry $[5,12]$. Compared with European registries, there is an increased female preponderance in the US population [37]. In relation to the older age at diagnosis, patients with $\mathrm{PAH}$ are more frequently obese and have more comorbidities such as hypertension, type II diabetes, ischaemic heart disease and atrial fibrillation $[5,10]$. In addition, differences in disease presentation appear in older patients. Symptoms at onset of the disease, such as peripheral oedema, are more often present in older patients compared with younger patients who experience more (near) syncope [10]. Furthermore, it seems that older patients have a less severe haemodynamic compromise, but a more limited functional capacity [10]. Most clinicians attribute the evolving epidemiology to improvements in referral patterns, increased PAH awareness, improvement in screening with echocardiography and unintentional inclusion of patients with $\mathrm{PH}$ due to heart failure with preserved ejection fraction, rather than a change in the disease phenotype itself $[4,10]$.

\section{The diagnostic delay of PAH}

Dyspnoea is the most frequent complaint at the onset of the disease for which patients seek medical attention. At the time of diagnosis, dyspnoea is the most pronounced symptom, combined with fatigue, angina, dizziness and oedema [4]. Due to the nonspecific nature of PAH symptoms, diagnosis of PAH is typically delayed. Unsurprisingly, delayed diagnosis potentially worsens prognosis of the disease [38]. In the 1980s, the NIH registry showed that patients were diagnosed approximately 2 years after the onset of the symptoms and most at a very advanced stage [4]. Unfortunately, awareness of PAH did not improve the diagnostic delay. The mean time between symptom onset and diagnosis in the French national registry data and in the REVEAL registry was 27 and 34 months, respectively [8, 9]. This delayed diagnosis is associated with a severe haemodynamic and functional profile. This issue was confirmed in the Spanish registry, in which $69 \%$ of the incident patients with PAH were in New York Heart Association functional class III or IV at diagnosis $[10,11]$. These results highlight the fact that adequate screening programmes should target patients with $\mathrm{PAH}$-associated conditions, in order to shorten the diagnostic delay. The best documented indication for screening is systemic sclerosis, but recommendations have been published for other subgroups [39].

\section{PH and left heart disease}

An important consequence of the increased awareness for PAH and age at diagnosis is the necessity to better differentiate IPAH from $\mathrm{PH}$ due to heart failure with preserved ejection fraction. $\mathrm{PH}$ related to left heart disease (LHD) represents by far the most common form of $\mathrm{PH}$, accounting for $65-80 \%$ of cases [40]. Proper distinction between $\mathrm{PAH}, \mathrm{PH}$ due to heart failure with preserved ejection fraction and other forms of PH-LHD may be challenging; however, the distinction has direct therapeutic consequences. An analysis of the COMPERA data showed that patients with atypical IPAH (more or equal to three risk factors for LHD) were older, more obese and had a lower exercise capacity but similar pulmonary haemodynamics, including PAWP, compared with typical IPAH (less than three risk factors for LHD). In addition, treatment effects were less pronounced in patients with $\mathrm{PH}$ due to heart failure with preserved ejection fraction compared with patients with atypical and typical IPAH [41]. Although PH-LHD is the most frequent form of $\mathrm{PH}$ in the population, most $\mathrm{PH}$ registries are currently not including patients with PH-LHD because, despite being referred, they are infrequently followed up in $\mathrm{PH}$ centres due to the absence of efficient therapies. 


\section{Treatment practices}

Taking into account that PAH remains an incurable condition with a poor prognosis, and based on clinical trial evidence, guidelines recommend a more aggressive treatment approach with the introduction of initial or sequential combination therapy for patients presenting with PAH [42]. However, recent registry data revealed that monotherapy was still used in the majority of patients. In the COMPERA registry, initial combinations of PAH drugs were only used in $17 \%$ of all patients within the first 3 months after diagnosis. At follow-up visits, between 3 months and 2 years after treatment initiation, this number increased towards $41 \%$. In the high-risk cohort, combination therapy was used in only $27 \%$ at baseline and $36 \%$ at follow-up [43], despite the recommendations to treat patients who have a high risk with combination therapy and parenteral prostacyclin therapy (supplementary table S1). In the Swedish registry, only $44 \%$ of the patients at high risk were receiving a combination of PAH drugs as first-line therapy (supplementary table S1) [44]. Likewise, only a minority of patients were treated with combination therapy in the ASPIRE registry [5, 13, 45]. Several factors might interfere with the results: the availability of PAH-specific drugs; the presence of side-effects; and the time-point at follow-up of data collection and analysis. Furthermore, these data might suggest that the use of combination therapy is less likely in elderly patients. A recent analysis of the Swedish registry data showed that elderly patients with IPAH (aged 6574 years and $\geqslant 75$ years) did not have improved risk profiles with treatment and confirmed that these patients were under-treated [46]. However, it is unknown whether elderly patients necessarily benefit less from an aggressive therapy approach.

Further investigations are needed to monitor whether: 1) the use of combination therapy will increase in the future; 2) this will be accompanied by better outcomes; and 3) to evaluate which therapy combinations are the most efficient and cost-effective.

\section{Oral anticoagulation}

The use of oral anticoagulation therapy in PAH was originally based on the observation of in situ thrombosis in the pulmonary circulation of patients with IPAH. Recent registries provided conflicting data. In the COMPERA registry, patients with IPAH treated with anticoagulation at any time $(92 \%$ within 3 months of diagnosis) had significantly improved survival compared with matched patients who never received oral anticoagulation therapy. No difference in survival was observed for patients with PAH-CTD [47]. In the REVEAL registry, patients with either IPAH or PAH-CTD, did not exhibit any benefit in survival [24]. Mortality was even increased in patients with PAH-CTD treated with warfarin within the previous year or any time post-baseline in comparison with warfarin-naïve patients. These conflicting data from two robust registries underscored the limitations of registry data but also the need for prospective RCTs on the use of oral anticoagulation therapy in PAH. An individual assessment should be made for each patient based on the aetiology of $\mathrm{PAH}$, severity, risk factors, comorbidities and medical history.

\section{Long-term survival/overall survival}

In 1984, the NIH registry was the first large registry to provide data on survival and prognosis of IPAH, HPAH and drug-induced PAH. The 1-, 3- and 5-year survival rates were 68\%, 48\% and 34\%, respectively [4]. Nowadays, in an era of multiple PAH therapies, registries show improved survival of $89-96 \%$ at 1 year and $73-77 \%$ at 3 years (figure 2). Patients with PAH associated with portal hypertension have the worse survival, followed by PAH-CTD, IPAH and PAH-CHD [11, 13, 51, 52]. In an ancillary study from the German National Register, survival rates in patients with Eisenmenger syndrome (PAH-CHD) are much lower than earlier reports had suggested, with survival rates among treatment-naïve patients of only $86 \%$, $60 \%$ and $34 \%$ at 1,5 and 10 years, respectively [53]. The single-centre Giessen PH Registry also reported similar, long-term transplant-free survival data from more than 2000 patients with different $\mathrm{PH}$ subtypes [52]. Moreover, the epidemiology and survival of patients with IPAH in Australia and New Zealand are similar to contemporary registries reported in Europe and North America [54]. In conclusion, there is an improving trend in the survival of $\mathrm{PAH}$ attributable to drug therapies, but the overall outcome of $\mathrm{PAH}$ still remains poor. A general observation is that most registries included a mixture of incident and prevalent patients, and that prevalent patients have a better long-term survival $[8,9]$. This is related to immortal time bias, which refers to a period of follow-up during which, by design, death cannot occur, meaning that prevalent patients with a poorer prognosis do not live long enough to be enrolled in a registry $[8,11,55]$. To take this into account, the UK registry included exclusively incident patients [10]. In the French registry, survival estimates and Cox proportional hazards model from time to diagnosis were adjusted for the bias arising from the delay between diagnosis and registry entry [55]. Patients were in the risk set only from their time of registry entry; for example, a patient recruited 1 year after diagnosis and followed-up for another 3 years was considered to enter the risk set at 12 months and was censored at 36 months (i.e. 24 months from study entry). 


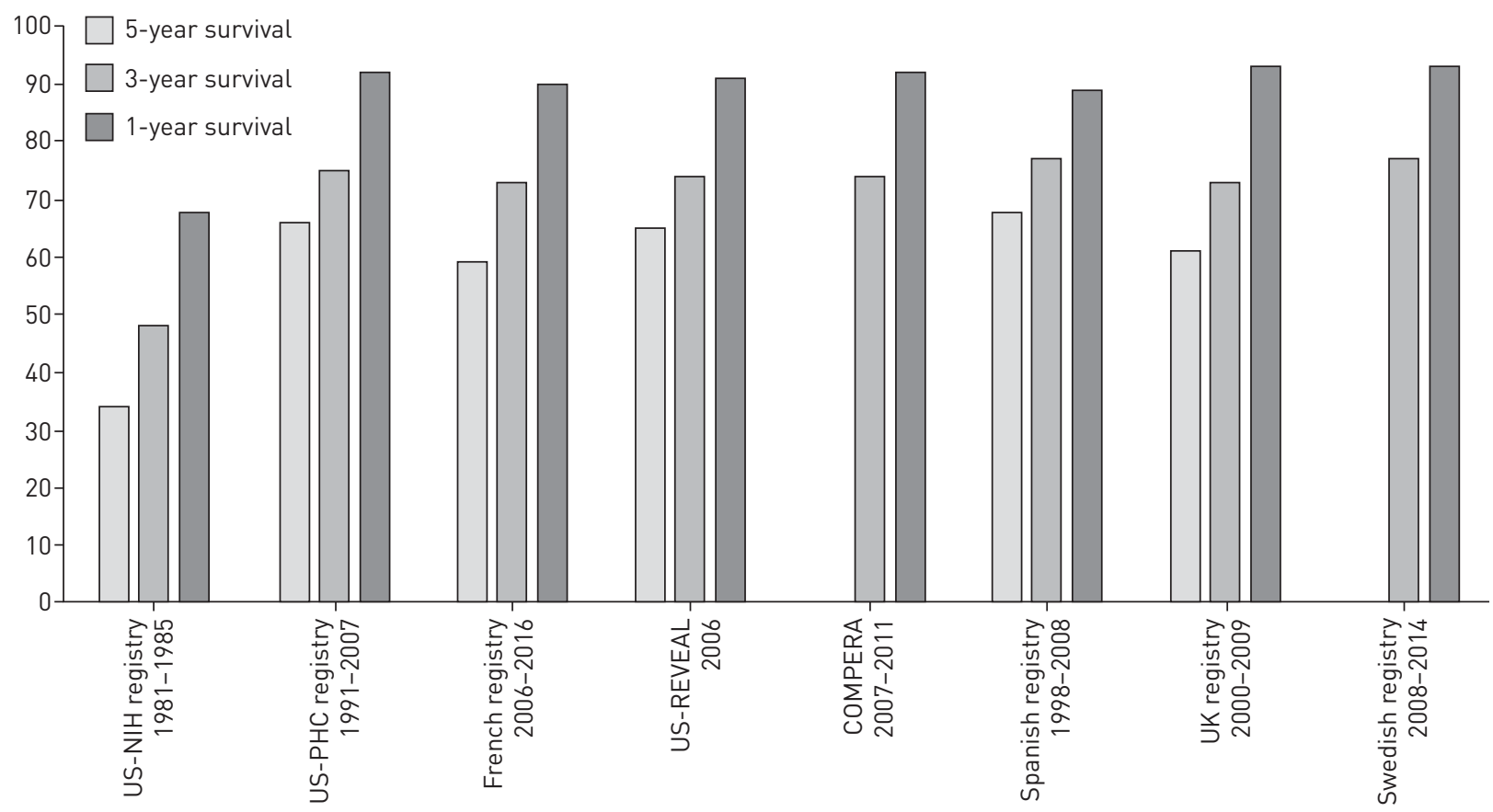

FIGURE 2 Pulmonary arterial hypertension (PAH) survival rates by subtypes and selected registries. US-NIH: United States National Institute of Health [7]; US-PHC: United States Pulmonary Hypertension Connection [48]; US-REVEAL: United States Registry to Evaluate Early and Long-Term Pulmonary Arterial Hypertension [9, 49]; COMPERA: Comparative, Prospective Registry of New Initiated Therapies for Pulmonary Hypertension [12]; Spanish registry [11]; UK registry [50]; Swedish registry [5].

\section{Risk prediction tools}

Ensuring optimal outcome requires prognostic evaluation and identifying individuals at risk at diagnosis and during follow-up in order to select the appropriate treatment in a timely manner. Regular risk assessments using multiple parameters are currently recommended by guidelines [42]. Registries have proven to be useful for the development of risk equations and risk scores. Currently, six prognostic equations or models have been proposed (table 3$)[6,7,42,50,54,56]$. The right atrial pressure and sex of the patient is included in almost all models as a predictor. Other parameters of prognostic relevance are patient demographics, signs and symptoms, functional class, exercise capacity assessed by the 6-min walk distance, echocardiography variables, cardiac magnetic resonance imaging variables, haemodynamic parameters and circulating brain natriuretic peptide (table 3). The first equation, based on data of the $\mathrm{NIH}$ registry, is outdated since the registry collected data before the availability of specific PAH drugs [7]. More recent equations aim to predict contemporary survival in idiopathic, heritable and drug-associated PAH $[50,51,55,56]$. The REVEAL risk score calculator and its updated version (REVEAL 2.0) and the equation of the French registry appeared accurate and well calibrated in validation cohorts, which confirms their generalisability $[55,57]$. Using the European Society of Cardiology/European Respiratory Society risk assessment approach, patients can be categorised as low, intermediate or high risk [42]. The risk assessment instrument has been validated at diagnosis and during follow-up in three registries: the Swedish registry, COMPERA and the French registry, which all demonstrated the benefit of reaching a low-risk profile $[43,44,58]$. Furthermore, the predictive value of haemodynamic variables, particularly the stroke volume index, and a multidimensional risk assessment was also recently demonstrated in incident patients with systemic sclerosis-associated PAH [59]. In addition, the use of an abbreviated version of the European Society of Cardiology/European Respiratory Society guideline risk assessment, previously proposed by BOUCLY et al. [58], was also accurate in predicting survival in incident patients with systemic sclerosis-associated PAH [60]. It was also shown that a combination of follow-up variables better predicted long-term prognosis compared with baseline variables and suggested that $\mathrm{N}$-terminal pro-brain natriuretic peptide was a reasonable surrogate for invasive haemodynamics [58, 61]. However, these approaches showed: 1) a limited ability to differentiate intermediate from high-risk patients; 2) that data collection was not standardised, with often significant missing data (except for the French registry that included only complete baseline and follow-up data); and 3) that some important prognostic features, such as imaging, echocardiography and a cardiopulmonary exercise test were not collected systematically in the registries. 
TABLE 3 Comparison of survival time and mortality prediction scores for pulmonary arterial hypertension (PAH)

[Ref.]

Risk assessment tools

Predictors of survival

Registry used for

\begin{tabular}{|c|c|c|c|c|c|}
\hline & & & & & \\
\hline \multirow{3}{*}{$\begin{array}{l}\text { NIH registry } \\
\text { PHC registry }\end{array}$} & [7] & IPAH & Risk equation & NYHA, RAP, PAP, Cl, $D_{\text {LCO }}$ & \\
\hline & [6] & $\mathrm{PAH}$ & & Age, CTD, NYHA, RAP, PAP, Cl & \\
\hline & & IPAH & Risk equation & Age, NYHA, RAP, PAP, Cl & \\
\hline \multirow[t]{2}{*}{ French registry } & {$[8]$} & $\mathrm{PAH}$ & & & REVEAL \\
\hline & & IPAH & Risk equation & Sex, 6MWD, CO & \\
\hline \multirow[t]{2}{*}{ REVEAL } & {$[46]$} & $\mathrm{PAH}$ & $\begin{array}{l}\text { Risk equation and risk } \\
\text { score table }\end{array}$ & $\begin{array}{c}\text { PVR, RAP, PO-PAH-FPAH-CTD, NYHA, sex, age, renal, BP, } \\
\text { HR, 6MWD, BNP, pericardial effusion, } D_{\text {LCo }}\end{array}$ & $\begin{array}{l}\text { French registry } \\
\text { UK registry } \\
\text { Spanish registry }\end{array}$ \\
\hline & & IPAH & & & \\
\hline Scottish/UK & {$[10]$} & $\begin{array}{l}\text { PAH } \\
\text { IPAH }\end{array}$ & Risk score & Age, sex, aetiology, 6MWD, RAP, CO & UK registry \\
\hline UK & {$[47]$} & & & & \\
\hline Spanish & {$[11]$} & $\begin{array}{l}\text { PAH } \\
\text { IPAH }\end{array}$ & & Sex, NYHA, RAP, CI, CTD-PO-PAH-PVOD & \\
\hline COMPERA & {$[12]$} & IPAH & & Sex, 6MWD & \\
\hline ESC/ERS & {$[36]$} & & Risk score table & & $\begin{array}{l}\text { Swedish registry } \\
\text { COMPERA registry } \\
\text { French registry }\end{array}$ \\
\hline $\begin{array}{l}\text { NIH: National In } \\
\text { Pulmonary Arter } \\
\text { ERS: European S } \\
\text { pressure; PAP: p } \\
\text { 6MWD: 6-min wa } \\
\text { pressure; HR: he }\end{array}$ & $\begin{array}{l}\text { Hyp } \\
\text { ety } \\
\text { non } \\
\text { ng }\end{array}$ & $\begin{array}{l}\text { Heal } \\
\text { ansio } \\
\text { ardi } \\
\text { arte } \\
\text { ance }\end{array}$ & $\begin{array}{l}\text { PHC: Pulmonary Hype } \\
\text { COMPERA: Comparative, } \\
\text { gy/European Respiratory } \\
\text { l pressure; Cl: cardiac it } \\
\text { O: cardiac output; PVR: } \\
\text { in natriuretic peptide; } \mathrm{P}\end{array}$ & $\begin{array}{l}\text { nsion Connection registry; REVEAL: Registry To Evaluate } \\
\text { rospective Registry of Newly Initiated Therapies for Pulmon } \\
\text { ociety; IPAH: idiopathic PAH; NYHA: New York Heart Associ } \\
\text { ex; } D_{\text {Lco: diffusion capacity for carbon monoxide; CTD: con }} \\
\text { Imonary vascular resistance; PO-PAH: porto-PAH; FPAH: } \\
\text { D: pulmonary veno-occlusive disease. }\end{array}$ & $\begin{array}{l}\text { arly and Long-Term } \\
\text { Hypertension; ESC/ } \\
\text { ion; RAP: right atrial } \\
\text { ctive tissue disease; } \\
\text { ilial PAH; BP: blood }\end{array}$ \\
\hline
\end{tabular}

validation

With this caveat in mind, we can conclude that the overall aim to compose risk stratifications based on easy-to-perform tests with no large burden for the patients was fulfilled.

\section{Benefits of registries}

$\mathrm{PAH}$ care is facing several issues, which can be addressed by investigation in clinical quality registries. First, a combination of disease registries with health service registries can provide insight in the increasing financial burden that results from an increasing prevalence of PAH and the introduction of advanced diagnostic technology and pharmaceutical therapies [62-64]. Secondly, pooling of cohort data can be a benefit for knowledge [65]. Thirdly, registries are the ideal tool to use for the determination of the best way of caring for patients, and they can be required for expert centres acquiring national accreditation [66]. Finally, observational studies are an important addition to RCTs for the detection of rare or late adverse effects of treatments, as shown for dasatinib, solvents, cyclophosphamide and mitomycin, which were shown to be risk factors for PAH or PVOD [67-71].

\section{Methodological considerations for future registries}

During the past decades, care for patients with PAH has been increasingly organised in specialised centres. Unfortunately, diagnoses are not systematically reported to national registries. However, this would greatly facilitate the determination of the incidence and prevalence of PAH in most countries. Currently, comparison of data should be performed with caution, given the existing heterogeneity between registries. There is a lack of harmonisation in data content across registries, for instance from one country to another or between/across registries with overlapping characteristics or patient cohorts. Acquiring data from different sources and pairing them in order to extract new knowledge is highly time consuming for researchers, healthcare providers organisations and policy makers. To avoid this, consensus on harmonised data structure for $\mathrm{PH}$ registries should be designed.

Recommendations in order to improve the quality of rare disease patient registries and data collection were developed in 2013 by the European Union (EU) Committee of Experts on Rare Diseases (EUCERD) [72]. First, the registries need to be internationally interoperable as much as possible and the data need to be harmonised and consistent. Secondly, all sources of data should be considered as sources of information and should be used for public health and research purposes. Thirdly, collected data should adhere to good practice guidelines and should be adaptable to serve regulatory purposes. Finally, the registries should be 
sustainable for a foreseeable timespan [72]. In the context of the EU's Health Programme 2008-2013, a cross-border patients registries initiative (PARENT) developed guidelines, which were created to provide practical and "hands on" advice to set up and manage patient registries as well as to enable secondary use of data for public health policy and research [3]. In addition, improving techniques which facilitate the sharing of data between registries should be developed. The latter is currently facilitated by use of common data elements in the design of new registries. This should ensure that data are defined in the same way, using the same standards and terminology [73].

In addition, one should secure the: 1) quality of the registry with confidence that design, conduct and analysis protects against bias (systematic errors) and errors in inference (i.e. erroneous conclusions drawn from registry data); and 2) quality, consistency and completeness of data entered into the database, with measures implemented to avoid errors of interpretation, in data entry and of intention with source document verification and site audits. Selection bias should be prevented by enrolment of all consecutive patients meeting inclusion criteria.

In May 2016, the EU adopted a new Regulation on the protection of personal data [74]. In practice, the enforcement of the General Data Protection Regulation (GDPR) might hamper the collection of medical data. For example, the GDPR introduced an informed consent that might impose researchers to ask for patient's specific consent, which might hinder the continuous research at every single use of the data. However, EU countries can individually set out the rules on a few key aspects of GDPR, including those on exemptions in scientific research. Consequently, the danger exists that data protection rules might not be the same across all EU countries. This can be very important to share precious data, and therefore slow down both research and useful innovation for patients. These initiatives are a first step towards greater interoperability of patient registries, although a number of complex challenges remain to be addressed.

\section{Conclusion}

Registries are useful tools to improve the comprehensive clinical evolution of PAH and provide data on epidemiology of PAH, characteristics of the PAH subgroups, demographics, symptoms, diagnostic delay, comorbidities, treatment practices, outcome predictors and risk stratification. Modern management registries should be further developed and extended, since there are many remaining unanswered questions, which can only be addressed in real-life settings. In the future, new trends and a systematic analysis of current PAH management should be further evaluated in pulmonary vascular medicine. Additionally, the influence of screening, emerging comorbidities or risk factors should be examined in future registries. Methodology should conform within current recommendations. Finally, the harmonisation of data collections and the reuse of data should be prioritised.

Conflict of interest: K. Swinnen has nothing to disclose. R. Quarck has nothing to disclose. L. Godinas reports personal fees from Actelion and MSD, outside the submitted work. C. Belge reports personal fees from Actelion and Bayer, outside the submitted work. M. Delcroix reports grants and personal fees from Actelion, personal fees from Bayer, GSK, MSD, Reata, Bellerophon and Eli Lilly, outside the submitted work.

\section{References}

1 Simonneau G, Montani D, Celermajer DS, et al. Haemodynamic definitions and updated clinical classification of pulmonary hypertension. Eur Respir J 2019; 53: 1801913.

2 McGoon MD, Benza RL, Escribano-Subias P, et al. Pulmonary arterial hypertension: epidemiology and registries. J Am Coll Cardiol 2013; 62: Suppl. 25, D51-D59.

3 Gliklich RE, Leavy MB, Karl J, et al. A framework for creating standardized outcome measures for patient registries. J Comp Eff Res 2014; 3: 473-480.

4 Rich S, Dantzker DR, Ayres SM, et al. Primary pulmonary hypertension. A national prospective study. Ann Intern Med 1987; 107: 216-223.

5 Rådegran $\mathrm{G}$, Kjellström B, Ekmehag $\mathrm{B}$, et al. Characteristics and survival of adult Swedish PAH and CTEPH patients 2000-2014. Scand Cardiovasc J SCJ 2016; 50: 243-250.

6 Thenappan T, Shah SJ, Rich S, et al. Survival in pulmonary arterial hypertension: a reappraisal of the NIH risk stratification equation. Eur Respir J 2010; 35: 1079-1087.

7 D’Alonzo GE, Barst RJ, Ayres SM, et al. Survival in patients with primary pulmonary hypertension. Results from a national prospective registry. Ann Intern Med 1991; 115: 343-349.

8 Humbert M, Sitbon O, Chaouat A, et al. Pulmonary arterial hypertension in France: results from a national registry. Am J Respir Crit Care Med 2006; 173: 1023-1030.

9 Badesch DB, Raskob GE, Elliott CG, et al. Pulmonary arterial hypertension: baseline characteristics from the REVEAL Registry. Chest 2010; 137: 376-387.

10 Ling Y, Johnson MK, Kiely DG, et al. Changing demographics, epidemiology, and survival of incident pulmonary arterial hypertension: results from the pulmonary hypertension registry of the United Kingdom and Ireland. Am J Respir Crit Care Med 2012; 186: 790-796.

11 Escribano-Subias P, Blanco I, López-Meseguer M, et al. Survival in pulmonary hypertension in Spain: insights from the Spanish registry. Eur Respir J 2012; 40: 596-603. 
12 Hoeper MM, Huscher D, Ghofrani HA, et al. Elderly patients diagnosed with idiopathic pulmonary arterial hypertension: results from the COMPERA registry. Int J Cardiol 2013; 168: 871-880.

13 Hurdman J, Condliffe R, Elliot CA, et al. ASPIRE registry: Assessing the Spectrum of Pulmonary hypertension Identified at a REferral centre. Eur Respir J 2012; 39: 945-955.

14 Humbert M, Segal ES, Kiely DG, et al. Results of European post-marketing surveillance of bosentan in pulmonary hypertension. Eur Respir J 2007; 30: 338-344.

15 Vachiéry J-L, Hoeper MM, Peacock AJ, et al. Ambrisentan use for pulmonary arterial hypertension in a post-authorization drug registry: the VOLibris Tracking Study. J Heart Lung Transplant 2017; 36: 399-406.

16 Klose H, Gall H, Ghofrani H-A, et al. Riociguat for the treatment of pulmonary hypertension: safety data from the EXPERT registry. Am J Respir Crit Care Med 2017; 1: A2292.

17 Abenhaim L, Moride Y, Brenot F, et al. Appetite-suppressant drugs and the risk of primary pulmonary hypertension. International Primary Pulmonary Hypertension Study Group. N Engl J Med 1996; 335: 609-616.

18 Manson JE, Faich GA. Pharmacotherapy for obesity - do the benefits outweigh the risks? N Engl J Med 1996; 335: 659-660.

19 Voelkel NF, Clarke WR, Higenbottam T. Obesity, dexfenfluramine and pulmonary hypertension. A lesson not learned? Am J Respir Crit Care Med 1997; 155: 786-788.

20 Channick RN, Simonneau G, Sitbon O, et al. Effects of the dual endothelin-receptor antagonist bosentan in patients with pulmonary hypertension: a randomised placebo-controlled study. Lancet 2001; 358: 1119-1123.

21 Rubin LJ, Black CM, Leconte I. Bosentan therapy for pulmonary arterial hypertension. N Engl J Med 2002; 346 : 896-903.

22 Kim NH, Brand M, Chin KM, et al. OPUS registry: safety and tolerability of macitentan in a real-world setting. J Heart Lung Transplant 2017; 36: S20-S21.

23 A registry for patients taking Uptravi (SPHERE). NCT03278002. https:/clinicaltrials.gov/ct2/show/NCT03278002 Date last updated: 11 September 2017; Date last accessed: 04 September 2019.

24 Preston IR, Roberts KE, Miller DP, et al. Effect of warfarin treatment on survival of patients with pulmonary arterial hypertension (PAH) in the Registry to Evaluate Early and Long-Term PAH Disease Management (REVEAL). Circulation 2015; 132: 2403-2411.

25 Peacock AJ, Murphy NF, McMurray JJV, et al. An epidemiological study of pulmonary arterial hypertension. Eur Respir J 2007; 30: 104-109.

26 Wijeratne DT, Lajkosz K, Brogly SB, et al. Increasing incidence and prevalence of World Health Organization groups 1 to 4 pulmonary hypertension: a population-based cohort study in Ontario, Canada. Circ Cardiovasc Qual Outcomes 2018; 11: e003973.

27 Mathai SC, Ryan Hemnes A, Manaker S, et al. Identifying patients with pulmonary arterial hypertension (PAH) using administrative claims algorithms. Ann Am Thorac Soc 2019; 16: 797-806.

28 Gillmeyer KR, Lee M-M, Link AP, et al. Accuracy of algorithms to identify pulmonary arterial hypertension in administrative data. Chest 2019; 155: 680-688.

29 van Riel ACMJ, Blok IM, Zwinderman AH, et al. Lifetime risk of pulmonary hypertension for all patients after shunt closure. J Am Coll Cardiol 2015; 66: 1084-1086.

30 Opravil M, Sereni D. Natural history of HIV-associated pulmonary arterial hypertension: trends in the HAART era. AIDS 2008; 22: Suppl. 3, S35-S40.

31 Sitbon O, Lascoux-Combe C, Delfraissy J-F, et al. Prevalence of HIV-related pulmonary arterial hypertension in the current antiretroviral therapy era. Am J Respir Crit Care Med 2008; 177: 108-113.

32 Monica L, Bruno D, Carlos J, et al. Cardiopulmonary manifestations of hepatosplenic schistosomiasis. Circulation 2009; 119: 1518-1523.

33 Papamatheakis DG, Mocumbi $\mathrm{AOH}$, Kim NH, et al. Schistosomiasis-associated pulmonary hypertension. Pulm Circ 2014; 4: 596-611.

34 Montani D, Lau E, Descatha A, et al. Pulmonary veno-occlusive disease as an occupational lung disease. Lancet Respir Med 2017; 5: e19.

35 Mandel J, Mark EJ, Hales CA. Pulmonary veno-occlusive disease. Am J Respir Crit Care Med 2000; 162: 1964-1973.

36 Al-Naamani N, Paulus JK, Roberts KE, et al. Racial and ethnic differences in pulmonary arterial hypertension. Pulm Circ 2017; 7: 793-796.

37 Frost AE, Badesch DB, Barst RJ, et al. The changing picture of patients with pulmonary arterial hypertension in the United States: how REVEAL differs from historic and non-US Contemporary Registries. Chest 2011; 139: 128-137.

38 Brown LM, Chen H, Halpern S, et al. Delay in recognition of pulmonary arterial hypertension. Chest 2011; 140: $19-26$.

39 Frost A, Badesch D, Gibbs JSR, et al. Diagnosis of pulmonary hypertension. Eur Respir J 2019; 53: 1801904.

40 Thenappan T, Gomberg-Maitland M. Epidemiology of pulmonary hypertension and right ventricular failure in left heart failure. Curr Heart Fail Rep 2014; 11: 428-435.

41 Opitz CF, Hoeper MM, Gibbs JSR, et al. Pre-capillary, combined, and post-capillary pulmonary hypertension: a pathophysiological continuum. J Am Coll Cardiol 2016; 68: 368-378.

42 Galiè N, Humbert M, Vachiery J-L, et al. 2015 ESC/ERS guidelines for the diagnosis and treatment of pulmonary hypertension: The Joint Task Force for the Diagnosis and Treatment of Pulmonary Hypertension of the European Society of Cardiology (ESC) and the European Respiratory Society (ERS): endorsed by: Association for European Paediatric and Congenital Cardiology (AEPC), International Society for Heart and Lung Transplantation (ISHLT). Eur Heart J 2016; 37: 67-119.

43 Hoeper MM, Kramer T, Pan Z, et al. Mortality in pulmonary arterial hypertension: prediction by the 2015 European pulmonary hypertension guidelines risk stratification model. Eur Respir J 2017; 50: 1700740.

44 Kylhammar D, Kjellström B, Hjalmarsson C, et al. A comprehensive risk stratification at early follow-up determines prognosis in pulmonary arterial hypertension. Eur Heart J 2018; 39: 4175-4181.

45 Sitbon O, Simonneau G. Optimal management of severe pulmonary arterial hypertension. Eur Respir Rev 2011; 20: $254-261$. 
46 Hjalmarsson C, Rådegran G, Kylhammar D, et al. Impact of age and comorbidity on risk stratification in idiopathic pulmonary arterial hypertension. Eur Respir J 2018; 51: 1702310.

47 Olsson KM, Delcroix M, Ghofrani HA, et al. Anticoagulation and survival in pulmonary arterial hypertension: results from the Comparative, Prospective Registry of Newly Initiated Therapies for Pulmonary Hypertension (COMPERA). Circulation 2014; 129: 57-65.

48 Thenappan T, Shah SJ, Rich S, et al. A USA-based registry for pulmonary arterial hypertension: $1982-2006$. Eur Respir J 2007; 30: 1103-1110.

49 Benza RL, Miller DP, Barst RJ, et al. An evaluation of long-term survival from time of diagnosis in pulmonary arterial hypertension from the REVEAL registry. Chest 2012; 142: 448-456.

50 Lee W-TN, Ling Y, Sheares KK, et al. Predicting survival in pulmonary arterial hypertension in the UK Eur Respir J 2012; 40: 604-611.

51 Benza RL, Gomberg-Maitland M, Miller DP, et al. The REVEAL registry risk score calculator in patients newly diagnosed with pulmonary arterial hypertension. Chest 2012; 141: 354-362.

52 Gall H, Felix JF, Schneck FK, et al. The Giessen pulmonary hypertension registry: survival in pulmonary hypertension subgroups. J Heart Lung Transplant 2017; 36: 957-967.

53 Diller G-P, Körten M-A, Bauer UMM, et al. Current therapy and outcome of Eisenmenger syndrome: data of the German National Register for congenital heart defects. Eur Heart J 2016; 37: 1449-1455.

54 Strange G, Lau EM, Giannoulatou E, et al. Survival of idiopathic pulmonary arterial hypertension patients in the modern era in Australia and New Zealand. Heart Lung Circ 2018; 27: 1368-1375.

55 Humbert M, Sitbon O, Chaouat A, et al. Survival in patients with idiopathic, familial and anorexigen-associated pulmonary arterial hypertension in the modern management era. Circulation 2010; 122: 156-163.

56 Benza RL, Miller DP, Gomberg-Maitland M, et al. Predicting survival in pulmonary arterial hypertension: insights from the Registry to Evaluate Early and Long-Term Pulmonary Arterial Hypertension Disease Management (REVEAL). Circulation 2010; 122: 164-172.

57 Benza RL, Gomberg-Maitland M, Elliott CG, et al. Predicting survival in patients with pulmonary arterial hypertension. The REVEAL risk score calculator 2.0 and comparison with ESC/ERS-based risk assessment strategies. Chest 2019; 156: 323-337.

58 Boucly A, Weatherald J, Savale L, et al. Risk assessment, prognosis and guideline implementation in pulmonary arterial hypertension. Eur Respir J 2017; 50: 1700889.

59 Weatherald J, Boucly A, Launay D, et al. Haemodynamics and serial risk assessment in systemic sclerosis associated pulmonary arterial hypertension. Eur Respir J 2018; 52: 1800678.

60 Mercurio V, Diab N, Peloquin G, et al. Risk assessment in scleroderma patients with newly diagnosed pulmonary arterial hypertension: application of the ESC/ERS risk prediction model. Eur Respir J 2018; 52: 1800497.

61 Nickel N, Golpon H, Greer M, et al. The prognostic impact of follow-up assessments in patients with idiopathic pulmonary arterial hypertension. Eur Respir J 2012; 39: 589-596.

62 Hoque DME, Kumari V, Hoque M, et al. Impact of clinical registries on quality of patient care and clinical outcomes: a systematic review. PLoS One 2017; 12: e0183667.

63 Burke JP, Hunsche E, Régulier E, et al. Characterizing pulmonary hypertension-related hospitalization costs among Medicare Advantage or commercially insured patients with pulmonary arterial hypertension: a retrospective database study. Am J Manag Care 2015; 21: Suppl. 3, s47-s58.

64 Sikirica M, Iorga SR, Bancroft T, et al. The economic burden of pulmonary arterial hypertension (PAH) in the US on payers and patients. BMC Health Serv Res 2014; 14: 676.

65 Kingswood JC, d'Augères GB, Belousova E, et al. TuberOus SClerosis registry to increase disease Awareness (TOSCA): baseline data on 2093 patients. Orphanet J Rare Dis 2017; 12: 2

66 Gliklich RE, Dreyer NA, Leavy MB, eds. Registries for evaluating patient outcomes: a user's guide. 3rd Edition. Agency for Healthcare Research and Quality. 2017.

67 Montani D, Bergot E, Günther S, et al. Pulmonary arterial hypertension in patients treated by dasatinib. Circulation 2012; 125: 2128-2137.

68 Montani D, Lau EM, Descatha A, et al. Occupational exposure to organic solvents: a risk factor for pulmonary veno-occlusive disease. Eur Respir J 2015; 46: 1721-1731.

69 Ranchoux B, Günther S, Quarck R, et al. Chemotherapy-induced pulmonary hypertension: role of alkylating agents. Am J Pathol 2015; 185: 356-371.

70 Perros F, Günther S, Ranchoux B, et al. Mitomycin-induced pulmonary veno-occlusive disease: evidence from human disease and animal models. Circulation 2015; 132: 834-847.

71 Belge C, Quarck R, Delcroix M, et al. Letter by Belge et al. regarding article, "Mitomycin-induced pulmonary veno-occlusive disease: evidence from human disease and animal models.”. Circulation 2016; 133: e591.

72 European Union Committee of Experts on Rare Diseases. 2013. EUCERD Core Recommendations on Rare Disease Patient Registration and Data Collection. Date last updated: 05 June 2013; Date last accessed: March 11, 2019. www.eucerd.eu/wp-content/uploads/2013/06/EUCERD_Recommendations_RDRegistryDataCollection_adopted.pdf

73 Sheehan J, Hirschfeld S, Foster E, et al. Improving the value of clinical research through the use of common data elements. Clin Trials 2016; 13: 671-676.

74 European Union. Regulation (EU) 2016/679 of the European Parliament and of the Council of 27 April 2016 on the protection of natural persons with regard to the processing of personal data and on the free movement of such data, and repealing Directive 95/46/EC (General Data Protection Regulation). https://publications.europa.eu/nl/ publication-detail/-/publication/3e485e15-11bd-11e6-ba9a-01aa75ed71a1/language-en Date last accessed: March 11, 2019. Date last updated: 2016. 